\title{
OBSERVACIONES SOBRE ACOLCHADO PLÁSTICO, IMIDACLOPRID Y HERRAMIENTAS DE IDENTIFICACIÓN DE MELANAGROMYZA TOMATERAE STEYSKAL (DIPTERA: AGROMYZIDAE) SOBRE PHYSALIS IXOCARPA BROT. (SOLANACEAE)
}

\author{
Rogelio Enrique PALACIOS TORRES, ${ }^{*}$ NÉSTOR BAUTISTA \\ MARTÍNEZ, ${ }^{1}$ José LuIS CARRILLO SÁNCHEZ, ${ }^{1}$ MIGuel ÁNGEL SÁNCHEZ \\ HERNÁNDEZ, ${ }^{2}$ BERTÍN MAURILIO JOAQUÍN TORRES ${ }^{2}$ \& JUAN FerNANDo \\ SOLÍS AGUILAR ${ }^{3}$ \\ *Autor para correspondencia: <rpalacios@unpa.edu.mx>, <rogeliopalaci57@hotmail.com> \\ ${ }^{1}$ Posgrado de Fitosanidad Entomología y Acarología. Colegio de Postgraduados, campus Montecillo, \\ Carr. México-Texcoco, km. 36.5, C. P. 56230. Montecillo, Texcoco, Estado de México, México. \\ ${ }^{2}$ Universidad del Papaloapan, campus Loma Bonita, Av. Ferrocarril S/N Col. Ciudad Universitaria. \\ C.P. 68400. Loma Bonita, Oaxaca, México. \\ ${ }^{3}$ Programa de Postgrado en Protección Vegetal, Departamento de Parasitología Agrícola. Universidad \\ Autónoma Chapingo, Km 38.5 Carr. México-Texcoco. C.P. 56230. Chapingo. Edo de México.
}

Palacios Torres, R. E., Bautista Martínez, N., Carrillo Sánchez, J. L., Sánchez Hernández, M. A., Joaquín Torres, B. M. \& Solís Aguilar, J. F. 2014. Observaciones sobre acolchado plástico, imidacloprid y herramientas de identificación de Melanagromyza tomaterae Steyskal (Diptera: Agromyzidae) sobre Physalis ixocarpa Brot. (Solanaceae). Acta Zoológica Mexicana (n.s.), 30(1): 61-73.

RESUMEN. En este estudio se evaluó el efecto de tres tipos de películas plásticas para acolchar el suelo: acolchado plástico transparente calibre $150(1.5 \mathrm{~mm})$, acolchado plástico negro/blanco calibre 125 $(1.25 \mathrm{~mm})$, acolchado plástico blanco/negro calibre $125(1.25 \mathrm{~mm})$ y el producto sistémico imidacloprid en dosis de 2.0 lt/ha sobre la presencia de estados inmaduros del barrenador del tallo Melanagromyza tomaterae, así como la respuesta productiva del cultivo de tomate de cáscara variedad CHF-1 a estos tratamientos. Se colectaron puparios y se colocaron en recipientes a temperatura ambiente para esperar la emergencia de adultos, los cuales se montaron y etiquetaron, los genitales del macho fueron extraídos para su estudio. Los resultados mostraron que este insecto prefiere infestar plantas vigorosas cultivadas en los plásticos al suelo, sin embargo esta preferencia no redujo el rendimiento de las plantas con mayor presencia del barrenador del tallo, ya que los rendimientos con acolchado plástico mostraron diferencias estadísticas significativas contrastando con los tratamientos sin acolchado plástico. La efectividad del imidacloprid se observó durante 65 días. Se proporcionan fotografías de los genitales del macho, así como una clave taxonómica para la identificación del barrenador del tallo del tomate de cáscara.

Palabras clave: Tomate de cáscara, genitalia del macho, Chapingo, Estado de México.

Recibido: 15/03/2013; aceptado: 04/10/2013. 
Palacios Torres, R. E., Bautista Martínez, N., Carrillo Sánchez, J. L., Sánchez Hernández, M. A., Joaquín Torres, B. M. \& Solís Aguilar, J. F. 2014. Observations on plastic mulching, imidacloprid and tools for the identification of Melanagromyza tomaterae Steyskal (Diptera: Agromyzidae) on Physalis ixocarpa Brot. (Solanaceae). Acta Zoológica Mexicana (n.s.), 30(1): 61-73.

ABSTRACT. The effect of three plastic film types for soil mulching: $1.5 \mathrm{~mm}$ clear plastic mulch, $1.25 \mathrm{~mm}$ black/white plastic mulch, and $1.25 \mathrm{~mm}$ white/black plastic mulch, and the application of the systemic product Imidacloprid at a dose of $2.0 \mathrm{~L} /$ ha over the presence of stem borer Melanagromyza tomaterae in its immature stages, as well as the crop yield response of tomatillo variety CHF-1 was evaluated. Pupae were collected and placed in containers at room temperature to await the emergence of adults, which were subsequently mounted and labeled, and the male genitals were removed for study. The results showed that this insect prefers infesting vigorous plants grown in plastics on the ground, but this preference did not reduce the yield of plants with greater borer presence, as yields with plastic mulch showed a significant statistical difference in contrast to the treatments without plastic mulch. The effectiveness of imidacloprid was observed along 65 days. A photograph of the male genitalia and a taxonomic key are provided for the identification of stem borers in tomatillo.

Key words: tomatillo, male genitals, Chapingo, The State of Mexico.

\section{INTRODUCCIÓN}

En México el tomate de cáscara (Physalis ixocarpa Brot.), llamado también tomate verde o tomatillo, es una hortaliza que se aprovecha desde épocas prehispánicas. Los aztecas lo cultivaban extensamente y lo utilizaban para confeccionar salsas y guisos de la misma manera como se emplea actualmente (Pérez et al.1997). Esta solanácea se cultiva todo el año tanto en el ciclo otoño-invierno como en primaveraverano. En el año 2010, la superficie total sembrada alcanzó las 48475 ha, en tanto que la superficie cosechada fue de 46197 ha obteniéndose una producción de 719 $848 \mathrm{t}$ con un rendimiento promedio de $15.58 \mathrm{t} \cdot \mathrm{ha}^{-1}$ (Anónimo 2010). Esta hortaliza es afectada por diferentes artrópodos que reducen su rendimiento y calidad. Uno de los insectos que en los últimos años ha despertado interés es el barrenador del tallo Melanagromyza tomaterae Steyskal (Diptera: Agromyzidae) llamado barrenador o arrocillo en las zonas productoras de jitomate y tomate de cáscara de América. Los primeros antecedentes sobre daños provocados por este díptero inician con su descripción taxonómica en plantas de jitomate Lycopersicon esculentum Miller en Colombia (Steyskal 1972). Algunas observaciones posteriores mencionan los efectos negativos de este barrenador sobre plantas de L. esculentum en Colombia, Ecuador y Venezuela (Spencer 1984; Havránek 1987; Probst et al. 1999; Montilla et al. 2007). En México este agromícido barrena los tallos del tomate de cáscara P. ixocarpa (Jiménez et al. 1992; Bautista \& Morales, 2000) y de algunas solanáceas silvestres tales como Jaltomata procumbens (Cav.) J. L. Gentry, Physalis patula Mill. (Palacios et al. 2008) y P. nicandroides Schltdl (Palacios et al. 2010). Su distribución abarca localidades de los estados de Hidalgo, México, Morelos, Puebla y Tlaxcala (Bautista \& Morales, 2000; Palacios et al. 2008; Palacios et al. 2010). Morales et al. (2007) mencionaron que la larva al alimentarse internamente del tallo daña los haces vascu- 
lares primarios, el parénquima y el xilema secundario causando los síntomas de amarillamiento y marchitez del vegetal, a su vez, Morales et al. (2002) indicaron que los daños favorecen la penetración e infección de la planta por el hongo Fusarium oxysporum Schlecht, que en conjunto reducen el rendimiento de esta hortaliza considerablemente.

Hasta la fecha, la información disponible sobre este díptero no proporciona elementos útiles para el desarrollo de sistemas de manejo de este insecto, solo se indica que los productores afectados utilizan insecticidas de manera irracional (Probst et al. 1999; Morales et al. 2002). Otras recomendaciones se limitan a indicar que la eliminación de malezas y residuos de cosecha pueden ayudar a disminuir su presencia en ciclos de cultivo posteriores (Morales et al. 2002; Montilla et al. 2007), por lo que es necesario documentar acciones sobre manejo de este insecto. Bajo este contexto se observó empíricamente durante el ciclo agrícola primavera-verano del año 1997, en Chapingo, Edo. de México la presencia de este barrenador sobre cultivos de tomate de cáscara sin acolchado y con acolchado plástico transparente, donde se observó que las plantas con películas plásticas mostraron cierta resistencia al barrenador. Por otro lado el producto sistémico imidacloprid al aplicarse al suelo (drench) cerca de la zona radicular provoca bajo impacto para la entomofauna benéfica, además por su alta residualidad dentro de la planta permite un control de una variedad de insectos que se traduce en pocas aplicaciones, lo cual representa ventajas para su empleo en programas de manejo integrado. A su vez, es conveniente contar con herramientas que faciliten una identificación taxonómica correcta, ya que este grupo de dípteros son difíciles de identificar y es necesario tener la certeza de la especie en estudio para poder documentar las estrategias de manejo sobre este agromícido. En consecuencia, en esta investigación, se evaluó la presencia de estados inmaduros de M. tomaterae sobre diferentes colores de acolchado plástico al suelo y el efecto de imidacloprid sobre éste díptero en el cultivo del tomate y se proporciona una clave para facilitar la identificación taxonómica de esta especie.

\section{MATERIAL Y MÉTODOS}

La presente investigación se realizó en condiciones de campo durante la primavera de 2002 en el Campo Experimental de la Universidad Autónoma de Chapingo, en Chapingo, Texcoco, Estado de México. Se efectuó una fase de laboratorio en el Instituto de Fitosanidad del Colegio de Postgraduados en Montecillo, Texcoco, Edo de México; ubicados entre $19^{\circ} 29^{\prime}$ de latitud norte y $98^{\circ} 53^{\prime}$ de longitud oeste y a una altura de 2250 metros sobre el nivel del mar. El clima de la localidad es del tipo $\mathrm{Cb}(\mathrm{Wo})(\mathrm{W})\left(\mathrm{i}^{\prime}\right) \mathrm{g}$, que corresponde a un clima templado subhúmedo con lluvias en verano, una época seca en invierno y con poca oscilación térmica $\left(5\right.$ y $\left.7{ }^{\circ} \mathrm{C}\right)$. La temperatura media anual es de $15.5^{\circ} \mathrm{C}$; mayo el mes más caliente y enero el más frío. La precipitación media anual es de $664 \mathrm{~mm}$ (López et al. 2009). 
Fase de campo. El experimento se llevó a cabo del 05 de abril (trasplante) al 28 de junio (cosecha) de 2002. La siembra del tomate de cáscara de la variedad CHF1-Chapingo, se realizó un mes antes del trasplante, se utilizaron charolas de poliestireno (unicel) de 200 cavidades, como sustrato de siembra se utilizó turba en su presentación mezcla 3 fina especial (SunShine ${ }^{\circledR}$ ). Las charolas permanecieron en invernadero donde se les suministró agua diariamente. Antes del trasplante se realizó una fertilización de fondo en el terreno definitivo con la formula 40-60-30 N-P-K. La colocación de las mangueras de riego y las películas plásticas del acolchado al suelo se realizaron después del surcado del terreno a una distancia de $1.20 \mathrm{~m}$, estas se colocaron manualmente sobre los surcos, la perforación del plástico se realizó con un tubo redondo a la distancia de $50 \mathrm{~cm}$. Se hicieron cuatro aplicaciones de 20N, 10P, 10K iniciando a los 15 días después del trasplante (ddt) y continuando cada 15 días hasta completar los cuatro suministros y la frecuencia de riego fue cada tercer día.

Diseño experimental y análisis estadístico. Se empleó un diseño experimental completamente al azar con cinco tratamientos y cuatro repeticiones, los tratamientos en estudio fueron: acolchado plástico transparente calibre $150(1.5 \mathrm{~mm})$, acolchado plástico negro/blanco calibre $125(1.25 \mathrm{~mm})$, acolchado plástico blanco/negro calibre 125 $(1.25 \mathrm{~mm})$, Imidacloprid, en dosis de $2.0 \mathrm{~L} \mathrm{ha}^{-1}$ aplicado al cuello de la planta a los 19 días después del trasplante, y un testigo absoluto. En cada fecha de muestreo, se seleccionaron cinco plantas al azar por unidad experimental las cuales se colocaron en bolsas grandes de plástico y se condujeron al laboratorio de Manejo Integrado de Plagas. Para obtener las variables de respuesta se promediaron los datos de las cinco plantas evaluadas por tratamiento en cada muestreo. El diámetro de tallo se tomó justo debajo de la horqueta y la altura de planta se midió desde el suelo hasta el dosel, en el caso de la presencia de insectos se inspeccionaron minuciosamente tallos y ramas de la planta para detectar cualquier instar larval, puparios vivos o muertos y puparios vacios del díptero, en el caso particular de esta variable, para su análisis estadístico, se sumó el total de insectos por tratamiento a partir del inicio de la presencia de insectos en las plantas del experimento que fue a los 48 días después del trasplante (ddt). A su vez para determinar la efectividad biológica del producto sistémico imidacloprid a partir del inicio de la presencia de insectos en el experimento, se revisaron dos plantas al azar hasta encontrar larvas dentro de los tallos para este tratamiento en particular. El rendimiento se cuantificó considerando el peso en kilogramos de las plantas que previamente se tomaron para obtener los datos de diámetro, altura e insectos presentes por planta.

El análisis de varianza se llevó a cabo con el procedimiento GLM, del paquete estadístico SAS ${ }^{\circledR}$ (SAS Institute 1999) y la comparación de medias se efectuó mediante la prueba de Tukey (Steel \& Torrie 1986). Previamente, la variable presencia de insectos se transformó con la función raíz cuadrada (Steel \& Torrie 1986) para estabilizar las varianzas. 
Identificación de Melanagromyza tomaterae. Para el montaje de los genitales del macho se separó el abdomen del resto del cuerpo del adulto. Posteriormente, este se maceró y se introdujo en una solución de $\mathrm{KOH}$ al $10 \%$, donde se dejó por cinco minutos a $80^{\circ} \mathrm{C}$ y después se enjuagó con agua destilada. Inmediatamente, se realizó otro lavado con agua destilada por 10 minutos a $80{ }^{\circ} \mathrm{C}$ aproximadamente (con el fin de eliminar el $\mathrm{KOH}$ restante en el tejido), como último paso se procedió a realizar la separación de la cutícula del abdomen, con pinzas de relojero, hasta dejar completamente limpios y expuestos los genitales. Todo el procedimiento se realizó utilizando una solución de alcohol al 70\%. Para el estudio e ilustración de las características de los genitales, se empleó un microscopio Tessovar de Carl Zeiss ${ }^{\circledR}$, un Fotomicroscopio III de Carl Zeiss ${ }^{\circledR}$ y una cámara digital para microscopia PAXcam $3^{\circledR}$ en donde la estructura se montó sobre un portaobjetos inmersa en gel transparente para cabello con glicerina, para que ésta se mantuviera en una posición estable.

Se emplearon los esquemas de la genitalia completa y edeago publicados por Steyskal (1972) y Spencer (1973), respectivamente con la finalidad de confirmar la identidad de la especie.

\section{RESULTADOS}

En cuanto a la morfología de la planta de tomate se registraron diferencias marcadas en las dos variables evaluadas. La altura mostró diferencias significativas $\left(F_{4,15}=\right.$ 17.86; $p=0.0001)$ en los tres muestreos realizados, siendo el plástico transparente el mejor tratamiento. En cuanto al diámetro del tallo sólo el primer muestreo presentó diferencias significativas entre tratamientos $\left(F_{4,15}=7.16 ; p=0.0020\right)$, donde el tratamiento con plástico negro/blanco generó el mayor desarrollo de diámetro, en el muestreo siguiente este mismo tratamiento fue numéricamente superior a los demás y para el último muestreo fue superior el tratamiento con imidacloprid sin alcanzar diferencias significativas (Cuadro 1).

La presencia de $M$. tomaterae se registró desde el 23 de mayo a los 48 días después del trasplante en el testigo absoluto y plástico negro/blanco. Al analizar el número promedio de larvas y puparios para todos los tratamientos no se registraron diferencias significativas entre ellos $\left(F_{4,15}=1.77 ; p=0.1866\right)$. El acolchado transparente mostró en promedio el doble de individuos respecto al negro/blanco. En el caso de los tratamientos testigo absoluto e imidacloprid se encontró que la presencia de estados inmaduros del díptero fue similar. El tratamiento con acolchado plástico blanco/negro tuvo la menor infestación (Cuadro 2). En el caso de la efectividad biológica del producto sistémico se observó la presencia de larvas hasta el 28 de junio, a los 65 días después de la aplicación del insecticida.

Las diferencias registradas entre los tratamientos en el rendimiento fueron altamente significativas $\left(F_{4,15}=19.78 ; p=0.0001\right)$ para el primer corte. El tratamiento con acolchado transparente, presentó el mayor rendimiento, los mejores resultados se 
Palacios Torres et al.: Manejo e identificación de Melanagromyza tomaterae

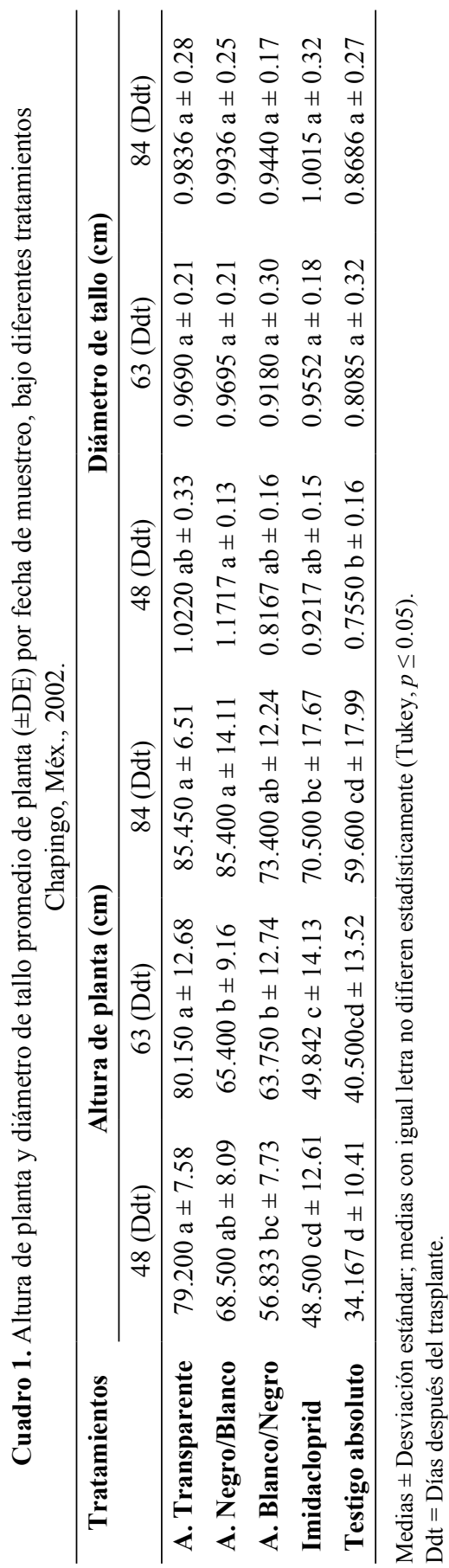


Cuadro 2. Número promedio ( $\pm \mathrm{DE}$ ) de larvas y/o puparios de $M$. tomaterae por planta, en las fechas de muestreo acumuladas bajo diferentes tratamientos en Chapingo, Méx., 2002.

\begin{tabular}{lc}
\hline \multicolumn{1}{c}{ Tratamientos } & Número promedio $( \pm$ DE) de larvas y/o puparios de M. tomaterae \\
\hline A. Transparente & $0.6725 \mathrm{a} \pm 0.36$ \\
A. Negro/Blanco & $0.3650 \mathrm{a} \pm 0.27$ \\
A. Blanco/Negro & $0.1148 \mathrm{a} \pm 0.09$ \\
Imidacloprid & $0.2880 \mathrm{a} \pm 0.38$ \\
Testigo absoluto & $0.2108 \mathrm{a} \pm 0.09$ \\
\hline
\end{tabular}

Medias \pm Desviación estándar; medias con igual letra no difieren estadísticamente (Tukey, $p \leq 0.05$ ).

registraron cuando los suelos fueron acolchados en comparación con los tratamientos a suelo desnudo. Para el segundo corte se detectaron diferencias marginales $\left(F_{4,15}\right.$ $=2.87 ; p=0.0596$ ), donde el tratamiento con plástico transparente fue el mejor superando por más de la mitad de su peso a los otros evaluados. Los rendimientos acumulados para los dos cortes muestran diferencias altamente significativas $\left(F_{4,15}=\right.$ $19.65 ; p=0.0001)$, indicando que el uso de acolchado plástico incrementa significativamente el rendimiento del cultivo de tomate de cáscara (Cuadro 3).

$\mathrm{Al}$ estudiar las características morfológicas de los genitales del macho de M. tomaterae se encontró que la parte anterior del epandrio es curvado con presencia de estructuras semejantes a uñas. El hipandrio, está formado por dos barras esclerosadas que se unen antes de su parte media conformando una sola estructura. El apodema edeagal presenta una longitud superior a $500 \mu \mathrm{m}$ en toda su longitud (Fig. 1A). El edeago en vista lateral presenta el distifalo con dos extensiones semejando una tenaza, donde la ubicada en la parte inferior es más ancha que la superior (Fig. 1 A y B). La bomba eyaculadora en su parte media está ensanchada y en su parte distal redondeada, con el centro bien definido (Fig. 1A). El distifalo en vista ventral no presenta

Cuadro 3. Rendimiento promedio $( \pm \mathrm{DE})$ obtenido en dos cortes en el experimento de tomate de cáscara bajo diferentes tratamientos, en Chapingo, Méx., 2002.

\begin{tabular}{lccc}
\hline \multicolumn{1}{c}{ Tratamientos } & $\mathbf{1}$ corte $\mathbf{6 3}(\mathbf{D d t})$ & $\mathbf{2}$ corte $\mathbf{8 4}(\mathbf{D d t})$ & Total \\
\hline A. Transparente & $3071.8 \mathrm{a} \pm 404.52$ & $537.3 \mathrm{a} \pm 347.11$ & $3609.1 \mathrm{a} \pm 473.52$ \\
A. Negro/Blanco & $1935.0 \mathrm{bc} \pm 426.31$ & $180.8 \mathrm{ab} \pm 145.80$ & $2115.8 \mathrm{bc} \pm 410.16$ \\
A. Blanco/Negro & $2194.0 \mathrm{ab} \pm 712.40$ & $251.8 \mathrm{ab} \pm 145.15$ & $2445.8 \mathrm{~b} \pm 821.58$ \\
Imidacloprid & $1012.5 \mathrm{~cd} \pm 299.13$ & $261.2 \mathrm{ab} \pm 204.69$ & $1273.7 \mathrm{~cd} \pm 430.84$ \\
Testigo absoluto & $538.5 \mathrm{~d} \pm 257.33$ & $71.4 \mathrm{~b} \pm 43.45$ & $609.9 \mathrm{~d} \pm 292.70$ \\
\hline
\end{tabular}

Medias \pm Desviación estándar; medias con igual letra no difieren estadísticamente (Tukey, $p \leq 0.05$ ).

Ddt $=$ Días después del trasplante 


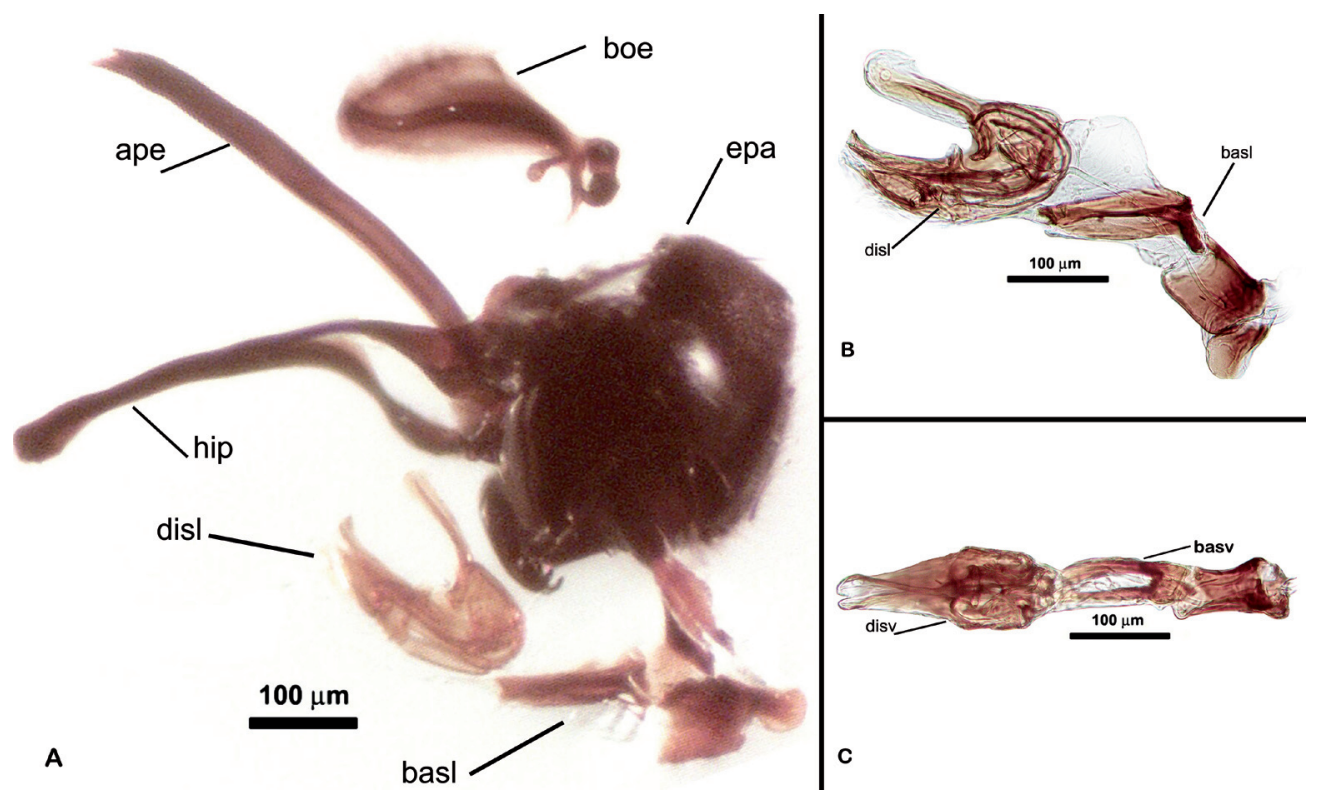

Figura 1. Genitales del macho de $M$. tomaterae Steyskal. A) Genitalia completa vista lateral. epa = Epandrio, hip $=$ Hipandrio, ape $=$ Apodema edeagal, disl $=$ Distifalo, basl $=$ Basifalo, boe $=$ Bomba eyaculadora. B) Edeago, vista lateral; disl = Distifalo, basl = Basifalo. C) Edeago, vista ventral; disv = Distifalo, basv $=$ Basifalo.

constricción, en su parte media es ancho y se hace angosto hacia su parte distal y los brazos del basifalo son alargados y unidos en sus dos extremos (Fig. 1C).

\section{DISCUSIÓN}

Varios estudios exponen las bondades que brinda el acolchado del suelo al emplearse en el cultivo del tomate de cáscara. Nuestros resultados confirman lo observado por Soldevilla et al. (2002) quienes indicaron que el uso de cubiertas de suelo modificó significativamente la morfología de las plantas, destacando el plástico transparente, ya que este favoreció el aumento del diámetro de tallo y altura de planta. Por su parte Morales \& Palacios (1998) concluyeron que el acolchado plástico transparente favorece significativamente el desarrollo de la planta de tomate de cáscara coincidiendo con los resultados obtenidos en este trabajo.

Diferentes estudios demuestran que los acolchados con plástico de color y transparente no tuvieron efecto en la disminución de las poblaciones de Liryomyza spp. (Diptera: Agromyzidae) en los cultivos de melón, jitomate y frijol (Webb \& Smith 1973; Price \& Poe 1976; Orozco et al. 1995). Los resultados del presente estudio muestran similitud, ya que la presencia de mayor cantidad de estados inmaduros de $M$. 
tomaterae también fue registrada sobre plantas bajo acolchado. Según Van Den Berg et al. (1998) las infestaciones del barrenador del tallo Melanagromyza sojae Zehntner estuvieron asociadas al mayor diámetro de tallo y altura de plantas en soya Glicine max (L.). Este comportamiento de elegir las plantas más vigorosas podría ser la causa de la mayor cantidad de estados inmaduros del barrenador encontrada en plantas de tomate de cáscara que se desarrollaron en acolchado plástico transparente.

El imidacloprid puede ser aplicado al follaje, al suelo o como tratamiento a la semilla (Tharp et al. 2000), algunos autores mencionan su efectividad contra muchas plagas chupadoras y raspadoras-chupadoras tales como áfidos, moscas blancas y trips (Powell \& Stoffella 1998; Marquini et al. 2002; Costa et al. 2011). Sin embargo, insectos con otros hábitos de alimentación como el masticador de las larvas que barrenan tallos, también son afectadas por este neonicotinoide, tal es el caso de Scirpophaga incertulas (Lepidoptera: Pyralidae) sobre el cultivo de arroz (Pandey \& Choubey 2012). Además, Morales et al. (2007) indicaron que las larvas de M. tomaterae se alimentan de los haces vasculares primarios, parénquima y xilema secundario, tejidos que son responsables del transporte de agua, nutrientes y sustancias elaboradas. Debido a este hábito los insectos tienen contacto con el producto sistémico dentro de tallos y ramas y son afectados hasta por 65 días aproximadamente después de su aplicación.

Los estudios realizados sobre tomate de cáscara $P$. ixocarpa han demostrado que los acolchados plásticos al suelo favorecen el desarrollo de la planta, conllevando un incremento en el rendimiento (Freyre \& Brent 2000; Soldevilla et al. 2002; López et al. 2009). El mejor tratamiento fue el acolchado con plástico transparente, que superó por casi seis veces al testigo absoluto que se cultivó sobre suelo desnudo. Esto coincide con lo reportado por Morales \& Palacios (1998) para tomate de cáscara donde el rendimiento de fruto bajo plástico transparente con dos cortes superó por $92.81 \%$ a lo obtenido en suelo desnudo. A su vez Velasco (1998) también encontró un incremento similar en el rendimiento del plástico transparente sobre el plástico negro en cuatro cortes. Por su cuenta Freyre \& Brent (2000) mostraron claramente el incremento del rendimiento de esta hortaliza bajo este sistema de producción.

Si bien Van Den Berg et al. (1998) indicaron que sólo existe una reducción de producción en el cultivo de soya G. max cuando la infestación de M. sojae es temprana, en el presente trabajo la aparición de insectos ocurrió en etapas avanzadas de desarrollo del cultivo, sin llegar a afectar los componentes del rendimiento.

Estos resultados aportan información con poblaciones bajas de M. tomaterae y ofrecen datos que sin lugar a dudas pueden ser tomados para valorar el comportamiento y daños y con este conocimiento implementar estrategias de manejo. Las cubiertas de plástico al suelo favorecen el vigor de la planta que no resiente la presencia de larvas barrenando tallos y ramas, ya que sus rendimientos son favorables. La dosis del imidacloprid utilizada en esta investigación puede tomarse como punto de referen- 
cia, ya que esta cantidad representa el doble de la dosis recomendada para hortalizas después del trasplante. En este sentido se requiere más investigación con el objetivo de evaluar dosis menores y aplicaciones tardías ya que la presencia del insecto en la zona es a finales de mayo. A su vez el empleo de los plásticos al suelo combinado con una adecuada y oportuna aplicación del producto sistémico pueden ser empleados en lugares donde este barrenador de tallos represente un problema. Las determinaciones taxonómicas correctas juegan un papel importante en el manejo de plagas, ya que con esta información se documentan con precisión acciones de manejo sobre la especie en particular y se generan antecedentes para próximas investigaciones.

El primer antecedente de la identificación de $M$. tomaterae en México fue erróneo ya que se ubicó en su momento en la familia Lonchaeidae (Jiménez et al. 1992). Las identificaciones taxonómicas erróneas son comunes, ya que en la literatura taxonómica antigua de Agromyzidae los dibujos representaban la única manera de interpretar las características distintivitas de cada especie y por otra parte la similitud morfológica externa entre especies cercanas crea dificultad para concluir taxonómicamente, así que en la mayoría de las veces la herramienta más contundente para obtener una identificación correcta, es a través del estudio de los genitales del macho. Las fotografías de la genitalía completa del macho y las vistas lateral y ventral del edeago de $M$. tomaterae pueden complementarse con los esquemas publicados por Steyskal (1972) y Spencer (1973), y con la clave para especies de Melanagromyza Hendel asociadas a plantas de interés agrícola para la correcta identificación de esta especie.

\section{Clave de identificación de especies de Melanagromyza Hendel (Diptera: Agromyzidae) que se alimentan de plantas de interés agrícola en México}

1. Especies con longitud alar menor a $2 \mathrm{~mm} \ldots \ldots \ldots \ldots \ldots \ldots \ldots \ldots \ldots \ldots \ldots \ldots \ldots \ldots$

1 '. Especies con longitud alar mayor a $2 \mathrm{~mm} \ldots \ldots \ldots \ldots \ldots \ldots \ldots$

2. Edeago en vista lateral con el basifalo y distifalo totalmente separados por cutícula membranosa, en vista ventral el distifalo presenta en su parte basal dos lóbulos, no presenta constricción y termina más angosto que el resto de éste. El basifalo en forma de "Y", separado del resto del edea-

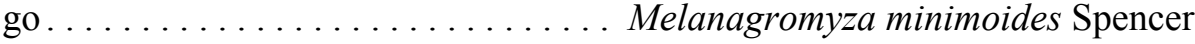

2'. Edeago en vista lateral con el distifalo que presenta en su parte superior una serie de puntos oscuros, además presenta una gran membrana cubriendo a todo el edeago. Edeago en vista ventral con el distifalo en su parte basal apenas más ancho que la distal, los brazos del basifalo forman

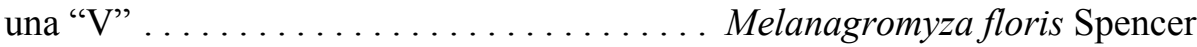

3. Coloración de mesonoto y abdomen verde-azulado. Edeago en vista lateral con el distifalo en su parte distal con dos extensiones asemejando una tenaza, donde la extensión baja es más ancha. Edeago en vista ventral donde el distifalo no presenta constricción, en su parte media es ancho y se hace angosto hacia 
su parte distal. Los brazos del basifalo alargados y unidos en sus dos extre-

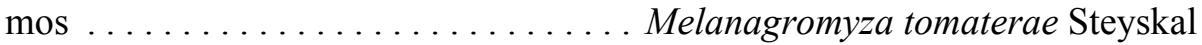

3'. Coloración de mesonoto y abdomen verde cobrizo . . . . . . . . . . 4

4. Órbitas ocelares ensanchadas en su parte media. Edeago en vista lateral con el distifalo en su parte distal baja se aprecian dos terminaciones separadas. Edeago en vista ventral con el distifalo sin constricción y termina con dos líneas medias separadas. Los brazos del basifalo forman una "U" bien definida con su base plana ................... Melanagromyza splendida Frick

4'. Órbitas ocelares sin ensancharse en su parte media . . . . . . . . . . . 5

5. Edeago en vista lateral con el distifalo curvo hacia arriba. Edeago en vista ventral con el distifalo presentando en su parte basal dos lóbulos pequeños, con una ligera constricción en su parte media. Los brazos del basifalo forma una "U" no muy bien marcada . . . . . . . . . Melanagromyza neotropica Spencer

5'. Edeago en vista lateral con el distifalo con extremos agudos dirigidos hacia arriba formando un ángulo casi recto. Edeago en vista ventral con el distifalo con una ligera constricción en su parte media y en su parte distal con dos extensiones paralelas pronunciadas. Los brazos del basifalo forman

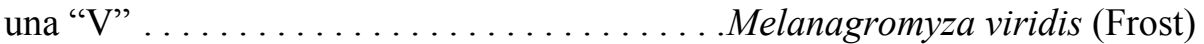

Agradecimientos. Al Consejo Nacional de Ciencia y Tecnología por la beca brindada al primer autor. Al Campo Experimental del Departamento de Fitotecnia de la Universidad Autónoma Chapingo por las facilidades otorgadas para la ejecución de la fase de campo. A su vez al Dr. Aureliano Peña Lomelí por su ayuda durante la ejecución del experimento. Al personal de campo del Colegio de Postgraduados y Universidad Autónoma Chapingo por su apoyo y a la Ingeniera en Diseño Carol Castro Reyes por su ayuda en la edición de las fotografías.

\section{LITERATURA CITADA}

Anónimo. 2010. Servicios de Información Agroalimentaria y Pesquera http://www.siap.gob.mx/index. php?option $=$ com_wrapper\&view $=$ wrapper\&Itemid $=35($ consultada 29/05/12) .

Bautista M., N. \& Morales G., O. 2000. Melanagromyza tomaterae Steyskal (Diptera: Agromyzidae) plaga del tomate (Physalis ixocarpaBrot. ) en México. Folia Entomológica Mexicana, 110: 129-130.

Costa, R. R., Moraes, J. C. \& DaCosta, R. R. 2011. Feeding behaviour of the greenbug Schizaphis graminum on wheat plants treated with imidacloprid and/or silicon. Journal of Applied. Entomology, 135: 115-120.

Freyre, R \& Brent. L., J. 2000. Evaluation and yield trials of tomatillo in New Hampshire. Hortechnology, 10: 374-377.

Havránek, D. 1987. Melanagromyza tomaterae (Diptera: Agromyzidae) a tomato pest in the States of Táchira and Mérida, Venezuela. Florida Entomologist, 70: 294-296.

Jiménez G., R., Domínguez R., R. \& Peña L., A. 1992. Plagas insectiles del tomate de cáscara (Physalis ixocarpa Brot.) en Chapingo, México. Revista Chapingo, 16: 75-79.

López, L. R., Arteaga R., R., Vázquez P., M. A.; López C., I. L., \& Sánchez C., I. 2009. Producción de tomate de cáscara (Physalis ixocarpa Brot.) basado en láminas de riego y acolchado plástico. Revista Chapingo Serie Horticultura, 15: 83-89. 
Marquini F. R., Guedes, N. C., Picanco, M. C. \& Regazzi, A. J. 2002. Response of arthropods associated with the canopy of common beans subjected to imidacloprid spraying. Journal of Applied Entomology, 126: 550-556.

Montilla, R., Lacruz, L., Durán, D., Medina, A. \& Ramos, F. 2007. Daños por Melanagromyza tomaterae, mosca barrenadora del tallo del tomate, en Trujillo, Venezuela. Agronomía Tropical, 57: 231-237.

Morales G., O., Bautista M., N., Valdez C., J. \& Carrillo S., J. L. 2002. Identificación, biología y descripción de Melanagromyza tomaterae Steyskal (Diptera: Agromyzidae), barrenador del tomate Physalis ixocarpa Brot. Acta Zoológica Mexicana (n.s.), 86: 145-153.

Morales R., J. \& Palacios T., R. E. 1998. Tomate de cáscara (Physalis ixocarpa Brot.) variedad CHF1- Chapingo en fertigación, bajo acolchado y fertilización foliar. Tesis de Licenciatura. Departamento de Fitotecnia. UACH. Chapingo, México. 84 pp.

Morales R, J., Yáñez J., P., Kohashi S., J. \& Bravo M., H. 2007. Estudio Anatómico del tallo de tomate de cáscara con daño por el barrenador (Melanagromyza tomaterae Steyskal). Agricultura Técnica en México, 33: 281-283.

Orozco, S. M., Pérez. Z., O. \& López A., O. 1995. Floating row cover and transparent mulch to reduce insect populations, virus diseases and increase yield in cantaloupe. The Florida Entomologist, 78: 493-501.

Palacios T. R. E., Romero N., J., Étienne, J., Carrillo S., J. L., Valdez C., J. M., Bravo M., H., Koch, S. D., López M., V. \& Terán V., A. P. 2008. Identificación, distribución y plantas hospederas de diez especies de Agromyzidae (Insecta: Diptera), de interés agronómico en México. Acta Zoológica Mexicana (n. s.), 24: 7-32.

Palacios T., R. E., Valdez, J. M., Étienne, J., Vega M.,R., Marín S., J., Castañeda V., A. \& Nava D., C. 2010. Nuevos registros de plantas hospederas y distribución geográfica de Melanagromyza floris Spencer, M. tomaterae Steyskal, M. viridis (Frost) y Ophiomyia lantanae (Froggatt) (Diptera: Agromyzidae) en México. Acta Zoológica Mexicana (n. s.), 26: 59-71.

Pandey, S. \& Choubey, M. N. 2012. Management of yellow stem borer, Scirpophaga incertulas in rice. Agricultura Science Digest, 32: 7-12.

Pérez G., M., Márquez S., F. \& Peña L., A. 1997. Mejoramiento genético de hortalizas. Primera edición. Departamento de Fitotecnia. UACH. Chapingo, Estado de México, México, 380 pp.

Powell, A. Ch. \& Stoffella, P. J. 1998. Control of tomato irregular ripening with Imidacloprid. HortScience, 33: 283-284.

Price, F. J. \& Poe, S. L. 1976. Response of Liriomyza (Diptera: Agromyzidae) and its parasites to stake and mulch culture of tomatoes. The Florida Entomologist, 59: 85-87.

Probst K., Pülschen, L., Sauerborn, J. \& Zebitz, C.P.W. 1999. Influencia de varios regímenes de uso de plaguicidas sobre la entomofauna de tomate en las tierras altas de Ecuador. Manejo Integrado de Plagas (Costa Rica), 54: 53-62.

SAS Institute. 1999. SAS/STAT. Software Version 8 for Windows. Cary, N.C27513, U.S.A.

Soldevilla., C. S., Peña L., A., Solís M., F., Vásquez R., T. R. \& Colinas L., M. T. 2002. Aplicación al suelo de $\mathrm{CO}_{2}$, uso de acolchados plásticos y sistemas de manejo en tomate de cáscara (Physalis ixocarpa Brot.). Revista Chapingo. Serie Horticultura, 8: 25-31.

Spencer, K. A. 1973. The Agromyzidae (Diptera) of Venezuela. Revista de la Facultad de Agronomía (Maracay), 7: 5-108.

Spencer, K. A. 1984. The Agromyzidae (Diptera) of Colombia, including a new species attacking potato in Bolivia. Revista Colombiana de Entomología, 10: 3-33.

Steel., R. G. D. \& Torrie, J. H. 1986. Bioestadística: principios y procedimientos. Segunda edición (primera en español) McGraw- Hill. México, D.F. 622 pp. 
Steyskal, G. C. 1972. Two new species of Melanagromyza Hendel (Diptera, Agromyzidae) that bore in tomato stalks in Colombia and Ecuador. Journal of the Washington Academy Sciences, 62: 265267.

Tharp, C., Blodgett, S. L. \& Johnson, G. D. 2000. Efficacy of imidacloprid for control of cereal leaf beetle (Coleoptera: Chrysomelidae) in barley. Journal Economic Entomology, 93: 38-42.

Van Den Berg, H., Shepard, B. M. \& Nasikin. 1998. Response of soybean to attack by stemfly Melanagromyza sojae in farmers fields in Indonesia. Journal of Applied Ecology, 35: 514-522.

Velasco G., V. 1998. Acolchado con película plástica obscura y transparente en la producción de tomate de cáscara (Physalis ixocarpa Brot) en sistema de riego por goteo y fertigación. Tesis de Licenciatura. Departamento de Fitotecnia. UACH. Chapingo, México. 48 pp.

Webb, E. R. \& Smith, F. F. 1973. Influence of reflective mulches on infestations of Liriomyza munda in snap bean foliage. Journal of Economic Entomology, 66: 539-540. 